\title{
CLASSIFICATION OF FINITE SIMPLICIAL ALGEBRAS
}

\author{
Alper ODABAŞ* \\ Department of Mathematics and Computer Sciences, Eskisehir Osmangazi University, \\ Art and Science Faculty, Eskisehir, Turkey
}

\begin{abstract}
We get a classification of 1-truncated simplicial algebras under certain conditions by using GAP
\end{abstract}

Keywords: Simplicial algebra, Moore complex, Crossed modules.

\section{INTRODUCTION}

Simplicial commutative algebras occupy a place somewhere between homological algebra, homotopy theory, algebraic K-theory and algebraic geometry. In each sector they have played a significant part in developments over quite a lengthy period of time. Their own internal structure has however been studied relatively little $[1,2]$.

The concept of a crossed module originates in the work [16] of Whitehead in algebraic topology. There the crossed modules were crossed modules of groups. Areas in which crossed modules have been applied include the theory of group presentations, algebraic K-theory, and homological algebra. The commutative algebra version of crossed modules has been used, in essence rather than in name, by Lichtenbaum and Schlessinger [12] also the work of Gerstenhaber [11] essentially involves the notion of crossed modules in commutative algebras. Some categorical results and Koszul complex link are also given by Porter in [14].

A share package XMod, [15], for the GAP computational discrete algebra system was described by C.D. Wensley et al. The 2-dimensional part of this programme contains functions for computing crossed modules and cat1-groups and their morphisms. Arvasi and Odabas describe a package XModAlg [6] for GAP4 which constructs crossed modules of k-algebras and cat1-algebras over $\mathrm{k}$, and their morphisms (see [7]).

By a similar way, we give a GAP implementation for classification of finite simplicial algebras. For this, we added some new functions which do not exist in XModAlg package. One of our main results is the GAP implementation of the equivalent categories crossed modules of algebras and that of simplicial algebras with Moore complex of length 1 .

\section{SIMPLICIAL ALGEBRAS}

In this section we recall a few well-known definitions and facts about simplicial algebras and homology modules. For more details regarding this, we refer to the book Homologie des algèbres commutatives by M.André [1]. Let $\mathbf{k}$ be a fixed commutative ring with $1 \neq 0 . E_{n}(n \in \mathrm{N})$ 
A simplicial algebra $\mathbf{E}$ is a collection of $\mathbf{k}$-algebras $E_{n}(n \in \mathbb{N})$ together with, for each $n \geq 0$, $\mathbf{k}$-algebra homomorphisms

$$
\begin{array}{cccc}
d_{i}^{n}: & E_{n} & \rightarrow E_{n-1} & 0 \leq i \leq n \neq 0 \\
s_{j}^{n}: & E_{n} & \rightarrow E_{n+1} & 0 \leq j \leq n
\end{array}
$$

which are called face operators and degeneracies respectively. These homomorphisms are required to satisfy the following axioms:

$$
\begin{array}{cll}
\text { 1. } & d_{i}^{n-1} d_{j}^{n}=d_{j-1}^{n-1} d_{i}^{n} & \text { for } 0 \leq i<j \leq n, \\
\text { 2. } & s_{i}^{n+1} s_{j}^{n}=s_{j+1}^{n+1} s_{i}^{n} & \text { for } 0 \leq i \leq j \leq n, \\
\text { 3. } & d_{i}^{n+1} s_{j}^{n}=s_{j-1}^{n-1} d_{i}^{n} & \text { for } 0 \leq i<j \leq n, \\
\text { 4. } & d_{i}^{n+1} s_{j}^{n}=\quad i d & \text { for } i=j \text { or } i=j+1, \\
\text { 5. } & d_{i}^{n+1} s_{j}^{n}=s_{j}^{n-1} d_{i-1}^{n} & \text { for } 0 \leq j<i-1 \leq n .
\end{array}
$$

A homomorphism of simplicial algebras $\boldsymbol{f}: \boldsymbol{E} \rightarrow \boldsymbol{F}$ is a set of $\mathbf{k}$-algebra homomorphisms $f_{n}$ : $E_{n} \rightarrow F_{n}$ commuting with all the face operators. We have thus defined the category of simplicial algebras, which we will denote by SimpAlg.

\subsection{The Moore Complex of a Simplicial Algebra}

Recall that given a simplicial algebra $\mathbf{E}$, the Moore complex (NE, $\partial$ ) of $\mathbf{E}$ is the chain complex defined by

with $\partial_{n}: N E_{n} \rightarrow N E_{n-1}$ induced from $d_{n}^{n}$ by restriction.

$$
(\mathbf{N E})_{n}=\bigcap_{i=0}^{n-1} \operatorname{Ker} d_{i}^{n}
$$

The $n^{\text {th }}$ homotopy module $\pi_{n}(\mathbf{E})$ of $\mathbf{E}$ is the $n^{\text {th }}$ homology of the Moore complex of $\mathbf{E}$, i.e.,

$$
\begin{aligned}
\pi_{n}(\mathbf{E}) & \cong \quad H_{n}(\mathbf{N E}, \partial) \\
& =\bigcap_{i=0}^{n} \operatorname{Ker} d_{i}^{n} / d_{n+1}^{n+1}\left(\bigcap_{i=0}^{n} \operatorname{Ker} d_{i}^{n+1}\right) .
\end{aligned}
$$

By a $k$-truncated simplicial algebra, we mean a simplicial algebra $\operatorname{tr}_{\mathbf{k}} \mathbf{E}$ obtained by forgetting dimensions of order $>k$ in a simplicial algebra $\mathbf{E}$. We denote the category of k-truncated simplicial algebras by $\operatorname{Tr}_{\mathbf{k}}$ SimpAlg. Recall from [9] some facts about the skeleton functor. In the category of algebras, $\mathbf{A l g}$, there is a truncation functor

which admits a right adjoint

$$
\operatorname{tr}_{\mathrm{k}}: \operatorname{SimpAlg} \rightarrow \operatorname{Tr}_{\mathrm{k}} \operatorname{SimpAlg}
$$

$$
\operatorname{cosk}_{\mathrm{k}}: \operatorname{Tr}_{\mathrm{k}} \operatorname{SimpAlg}_{\rightarrow} \text { SimpAlg }
$$

called the $k$-coskeleton functor, and a left adjoint

called the $k$-skeleton functor.

$$
\mathbf{s k}_{\mathrm{k}}: \operatorname{Tr}_{\mathrm{k}} \operatorname{SimpAlg} \rightarrow \text { SimpAlg }
$$




\section{CROSSED MODULES}

J.H.C.Whitehead [16] described crossed modules in various contexts especially in his investigations into the algebraic structure of relative homotopy groups. In this section, we introduce the definition and elementary theory of crossed modules of commutative algebras given by T.Porter, [14]. More details about this may be found in $[3,4,5]$.

Let $R$ be a k-algebra with identity. A pre-crossed module of commutative algebras is an $R$-algebra $C$, together with a commutative action of $R$ on $C$ and an $R$-algebra morphism

such that for all $c \in C, r \in R$

$$
\partial: C \rightarrow R,
$$

$$
\text { CM1) } \partial(r \cdot c)=r \partial c \text {. }
$$

This is a crossed R-module if in addition, for all $c, c^{\prime} \in C$,

$$
\text { CM2) } \partial c \cdot c^{\prime}=c c^{\prime} .
$$

The last condition is called the Peiffer identity. We denote such a crossed module by $(C, R, \partial)$. Clearly any crossed module is a pre-crossed module.

A morphism of crossed modules from $(C, R, \partial)$ to $\left(C^{\prime}, R^{\prime}, \partial^{\prime}\right)$ is a pair of k-algebra morphisms,

$$
\theta: C \rightarrow C^{\prime}, \psi: R \rightarrow R^{\prime},
$$

such that

$$
\theta(r \cdot c)=\psi(r) \cdot \theta(c) \text { and } \partial^{\prime} \theta(c)=\psi \partial(c)
$$

In this case, we shall say that $\theta$ is a crossed R-module morphism if $R=R^{\prime}$ and $\psi$ is the identity. We therefore can define the category of crossed modules denoting it as XMod.

\section{Examples}

1. Let I be any ideal of a $\mathbf{k}$-algebra $\mathrm{R}$. Consider an inclusion map

$$
\text { inc. }: I \rightarrow R \text {. }
$$

Then $(I, R$, inc. $)$ is a crossed module. Conversely given any crossed R-module $\partial: C \rightarrow R$, one can easily verify that $\partial C=I$ is an ideal in $\mathrm{R}$.

2. Let $\mathrm{M}$ be any R-module. It can be considered as an R-algebra with zero multiplication, and then $\mathbf{0}: M \rightarrow R$ is a crossed R-module by $\mathbf{0}(c) \cdot c^{\prime}=0 c^{\prime}=0=c c^{\prime}$, for all $c, c^{\prime} \in C$.

Conversely, given any crossed modüle $\partial: C \rightarrow R$, then $\operatorname{Ker} \partial$ is an $R / \partial C-$ module.

3. Assume given a simplicial algebra $\mathbf{E}$ and a simplicial ideal $\mathbf{I}$. The inclusion

induces a map

$$
\text { inc. : } \mathbf{I} \hookrightarrow \mathbf{E} \text {, }
$$




$$
\partial: \pi_{0}(\mathbf{I}) \rightarrow \pi_{0}(\mathbf{E})
$$

and $\mathbf{E}$ acting on $\mathbf{I}$ by multiplication induces an action of $\pi_{0}(\mathbf{E})$ on $\pi_{0}(\mathbf{I})$. Then $\left(\pi_{0}(\mathbf{I}), \pi_{0}(\mathbf{E}), \partial\right)$ is a crossed module.

Proposition 1 If $(C, R, \partial)$ is a crossed $R$-module, then

i) Ker $\partial$ is a central ideal of $C$,

ii) both $C / C^{2}$ and Ker $\partial$ have natural $R / \partial C$-module structure (See [13]).

Theorem 2 The category of crossed modules is equivalent to the category of simplicial algebras with Moore complex of length 1. (See Arvasi and Porter, [3])

Proof. Let $\mathbf{E}$ be a simplicial algebra with Moore complex of length 1. Put $M=N E_{1}, N=N E_{0}$ and $\partial_{1}=$ $d_{1}$ (restricted to $M$ ). Then $N E_{0}$ acts on $N E_{1}$ by multiplication via $s_{0}$. Since the Moore complex is of length 1 , we have $\partial_{2} N E_{2}=\operatorname{Kerd}_{0} \operatorname{Kerd}_{1}=0$ and the generators of this ideal are of the form $x\left(s_{0} d_{1} y-y\right)$ with $x, y \in N E_{1}$. It then follows that for all $x, x^{\prime} \in M$,

$$
\begin{aligned}
& \partial_{1}(x) \cdot x^{\prime}=d_{1}(x) \cdot x^{\prime} \\
& =s_{0} d_{1}(x) x^{\prime} \quad \text { by the action, } \\
& =x x^{\prime} \quad \text { since } \partial_{2} N E_{2}=0 \text {. }
\end{aligned}
$$

Thus $\partial_{1}: M \rightarrow N$ is a crossed module. This yields a functor

$$
\mathbf{N}_{1}: \text { SimpAlg } \leq 1 \rightarrow \text { XMod }
$$

Conversely, let $\partial_{1}: M \rightarrow N$ be a crossed module. By using the action of $N$ on $M$, one forms the semidirect product $M \rtimes N$ together with homomorphisms

$$
d_{0}(m, n)=n, d_{1}(m, n)=\partial_{1} m+n, s_{0}(n)=(0, n) .
$$

Define $E_{0}=N$ and $E_{1}=M \rtimes N$. Then we have a 1-truncated simplicial algebra $\mathbf{E}_{\leq 1}$.

There is a functor $t_{1]}$ from the category of 1-truncated simplicial algebras to that of simplicial algebras. This enables us to define a functor

$$
\text { XMod } \rightarrow \operatorname{SimpAlg}_{\leq 1}
$$

given by sending $\{M, N, \partial\}$ to $\mathbf{E}=t_{1]} \mathbf{E}_{\leq \mathbf{1}}$. $\mathbf{E}$ is a simplicial algebra whose Moore complex is of length 1 . The correspondence gives rise to an equivalence of categories.

\section{APPLICATIONS}

GAP [10] is an open-source system for discrete computational algebra. The system consists of a library of implementations of mathematical structures: groups, vector spaces, modules, algebras, graphs, codes, designs, etc.; plus databases of groups of small order, character tables, etc. The system has world wide usage in the area of education and scientific research. GAP is free software and user contributions to the system are supported. These contributions are organized in a form of GAP packages and are distributed together with the system. Contributors can submit additional packages for inclusion after a reviewing process. 
The Small Groups library provides access to descriptions of the groups of small order up to isomorphism. There is no equivalent library of small algebras in GAP. For (commutative) algebras, we will concentrate on group rings of abelian groups over finite fields, because these algebras are conveniently implemented in GAP. We recall some basic properties of group algebras as follows.

Group Algebras : Let $\mathbf{k}$ be a field and $G$ a multiplicative group, finite or infinite. It is well known that the group algebra $\mathbf{k} G$ is an associative $\mathbf{k}$-algebra with a set $\left\{e_{g}: g \in G\right\}$ as a basis and with multiplication defined distributively using the group multiplication in $G$.

For $\sigma: G \rightarrow H$ a group homomorphism, let the map $\mathbf{k} \sigma$ be the group algebra homomorphism where:

$$
\begin{aligned}
\boldsymbol{k} \sigma: \boldsymbol{k} G & \rightarrow \boldsymbol{k} H \\
e_{g} & \mapsto e_{\sigma(g) .}
\end{aligned}
$$

In particular $\mathbf{k i d}_{G}=\mathrm{id}_{\mathbf{k} G}$, and if $\sigma^{\prime}: H \rightarrow J$ is a second group homomorphism, then $\mathbf{k}\left(\sigma * \sigma^{\prime}\right)=\mathbf{k} \sigma *$ $\mathbf{k} \sigma^{\prime}$. These facts are summarized in the following proposition (see [8]).

Proposition 3 k(.): Gr $\rightarrow$ Alg is a functor.

The group algebra functor provides a canonical construction for $\mathbf{k}$-algebra from any given group. Conversely, there are at least two canonical ways of extracting a group from a given $\mathbf{k}$-algebra. One is to forget the multiplication and take the additive (abelian) group of the algebra; this gives the forgetful functor Alg $\rightarrow \mathbf{A b}$. Alternatively, the subset of the algebra consisting of elements which are invertible under multiplication forms a subgroup (with the operation of multiplication) called the group of units of the algebra; this gives a functor $\mathbf{u}():. \mathbf{A l g} \rightarrow \mathbf{G r}$. In general, the group of units of a non-commutative algebra need not be abelian.

Proposition 4 The group algebra functor $\mathbf{k}():. \mathbf{G r} \rightarrow \mathbf{A l g}$ is left adjoint to the unit group functor $\mathbf{u}():$. Alg $\rightarrow$ Gr.

Proof. Let $G$ be a group and $A$ a k-algebra, and suppose $f: G \rightarrow u(A)$. Define a map $\omega_{G, A}: \operatorname{Gr}(G, u(A)) \rightarrow \operatorname{Alg}(\mathbf{k} G, A)$ by

$$
\omega_{G, A}(f)\left(\mathbf{e}_{g}\right):=\mathbf{e}_{f(g)}
$$

(this defines $\omega_{G, A}$ completely, since $\left\{\mathbf{e}_{g}: g \in G\right\}$ is a basis for $\mathbf{k} G$ and, for every $g \in G, \omega_{G, A}(f)\left(\mathbf{e}_{g}\right) \in$ $\mathbf{k} u(A) \subseteq A)$.

Suppose $\psi: \mathbf{k} G \rightarrow A$. Then $\psi$ is completely determined by $\left\{\psi\left(\mathbf{e}_{g}\right): g \in G\right\}$, and for each $g \in G$

$$
1_{A}=\psi\left(\mathbf{e}_{g} \mathbf{e}_{g^{-1}}\right)=\psi\left(\mathbf{e}_{g}\right) \psi\left(\mathbf{e}_{g^{-1}}\right)
$$

so $\psi\left(\mathbf{e}_{g}\right) \in u(A)$. Define the map $\varpi_{G, A}: \operatorname{Alg}(\mathbf{k} G, A) \rightarrow \mathbf{G r}(G, u(A))$ by

$$
\varpi_{G, A}(\psi) g=\psi\left(\mathbf{e}_{g}\right) .
$$

Now $\left[\varpi_{G, A} \omega_{G, A}(f)\right](g)$ and $\left[\omega_{G, A} \varpi_{G, A}(\psi)\right](g)=\psi(g)$, so $\omega_{G, A}$ is a bijection and

$$
\operatorname{Gr}(G, u(A)) \cong \mathbf{A} \lg (\mathbf{k} G, A)
$$


as required. It is easy to see that $\omega$ and $\varpi$ are natural in $G$ and $A$. The remaining cases are proved similarly (see [8]).

Remark : The functor $\mathbf{k}($.$) does not extend to actions and semidirect products. In particular, an action$ of a group $R$ on a group $S$ does not extend naturally to an action of $\mathbf{k} R$ on $\mathbf{k} S$.

We have developed functions for GAP4 which construct simplicial algebras. Functions to construct simplicial algebras

SimplicialAlgebra,

SimplicialAlgebraTr1ByFaceDegenere, SimplicialAlgebraTr1ByEndomorphisms, SimplicialAlgebraTr1, IsSimplicialAlgebraTr1 and IsSimplicialAlgebra. Attributes of a simplicial algebra constructed in this way include Source, Range, Face, Degenere, Size and Name.

In the following GAP session, we construct a simplicial algebra by using the group algebra $G F_{2} C_{6}$ and $G F_{2} C_{3}$. Also we show usage of the attributes listed above.

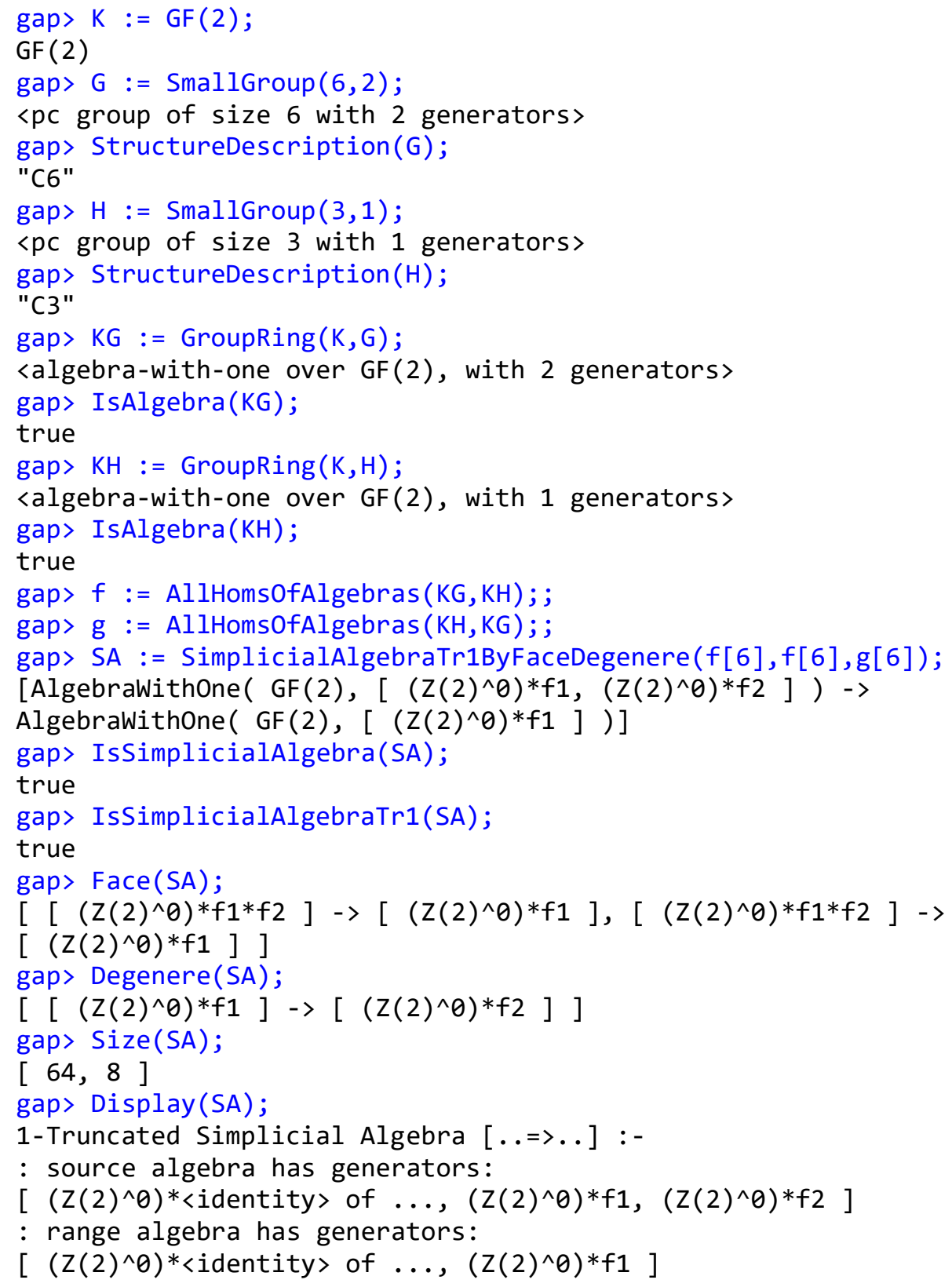


: face operator d10 maps source generators to:

$\left[\left(Z(2)^{\wedge} \theta\right)^{*}\langle\right.$ identity $\rangle$ of $\ldots,\left(Z(2)^{\wedge} \theta\right)^{*}\langle$ identity $\rangle$ of $\ldots$,

$\left.\left(Z(2)^{\wedge} \theta\right) * f 1\right]$

: face operator d11 maps source generators to:

$\left[\left(Z(2)^{\wedge} \theta\right)^{*}\left\langle\right.\right.$ identity $>$ of $\ldots,\left(Z(2)^{\wedge} \theta\right)^{*}\langle$ identity $\rangle$ of ...,

$\left.\left(Z(2)^{\wedge} \theta\right) * f 1\right]$

: degenere operator s00 maps range generators to:

$\left[\left(Z(2)^{\wedge} \theta\right) *<\right.$ identity> of $\left.\ldots,\left(Z(2)^{\wedge} \theta\right) * f 2\right]$

We have developed the function AllSimplicialAlgebrasTr1 which constructs all 1-truncated simplicial algebras. On the other hand, the function IsMooreComplex1 is used to verify the Moore complex of length 1.

In the following GAP session, we construct all 248 1-truncated simplicial algebras by using the group algebra $\mathrm{GF}_{3} \mathrm{Kl}_{4} .25$ of all 248 1-truncated simplicial algebras are Moore complex of length 1.

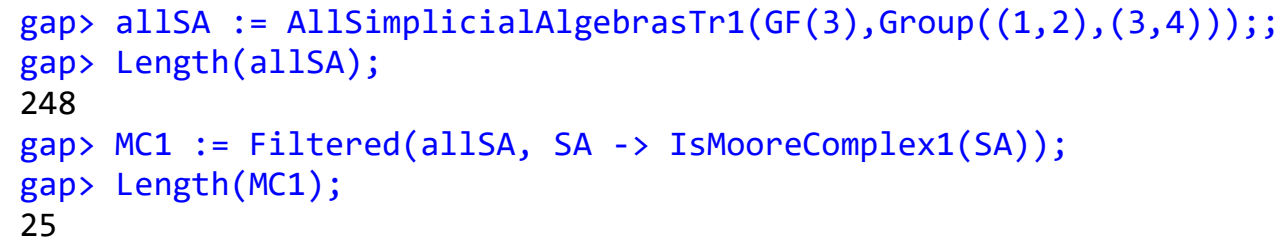

By using the natural equivalence of categories of crossed modules and the category of simplicial algebras with Moore complex of length 1, we have developed the functions XModAlgebraBySimplicialAlgebra and SimplicialAlgebraByXModAlgebra which constructs crossed modules and simplicial algebras from the given simplicial algebras and crossed modules, respectively.

In the following GAP session, we get a crossed module from a simplicial algebras with Moore complex of length 1 .

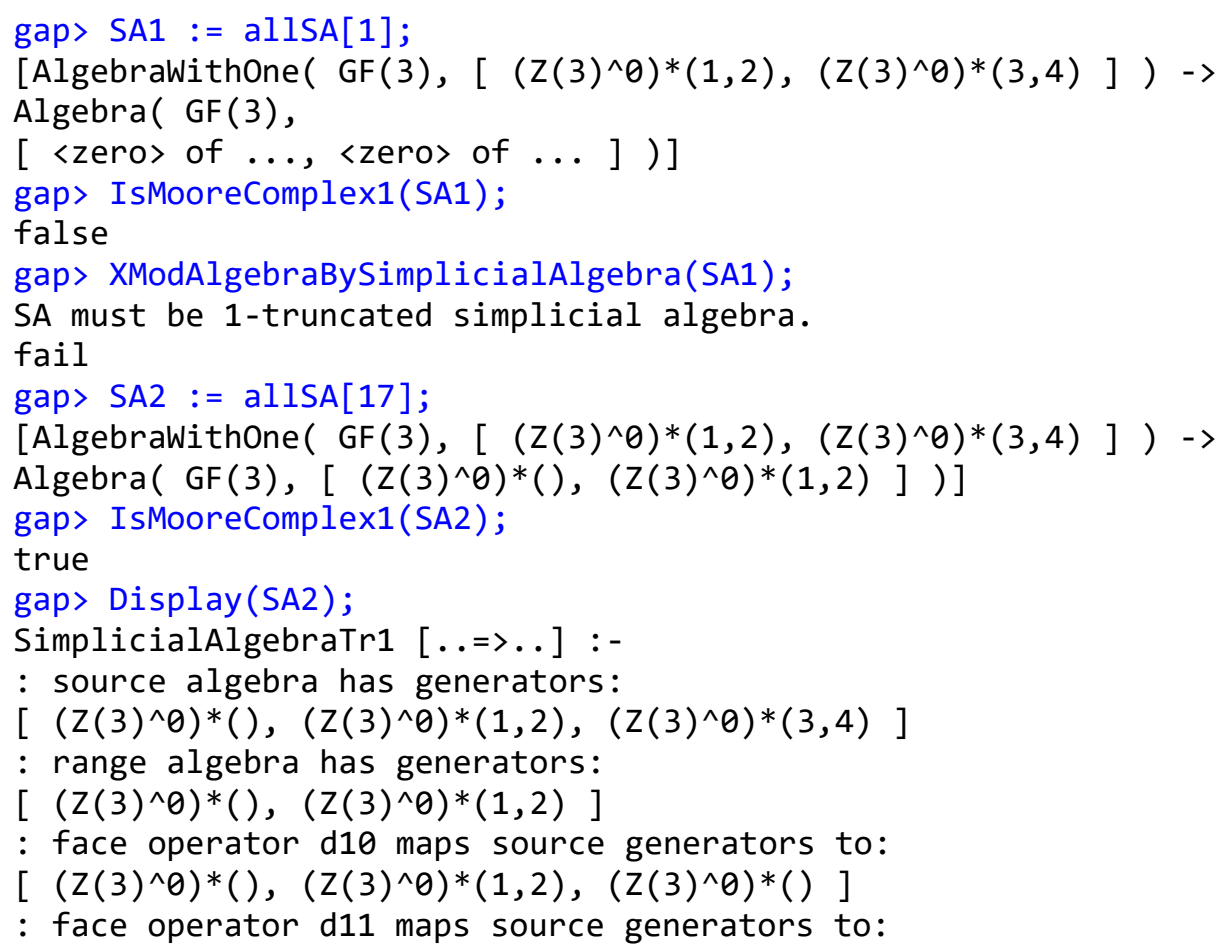


$\left[\left(Z(3)^{\wedge} \theta\right) *(),\left(Z(3)^{\wedge} \theta\right) *(1,2),(Z(3)) *()\right]$

: degenere operator s00 maps range generators to:

$\left[\left(Z(3)^{\wedge} \theta\right) *(),\left(Z(3)^{\wedge} \theta\right) *(1,2)\right]$

gap> Size $(\mathrm{SA} 2)$;

$[81,9]$

gap> CM := XModAlgebraBySimplicialAlgebra(SA2);

[Algebra( $\mathrm{GF}(3),\left[\left(Z(3)^{\wedge} \theta\right) *()+\left(Z(3)^{\wedge} \theta\right) *(3,4)\right.$,

$\left.\left.\left(Z(3)^{\wedge} \theta\right) *(1,2)+\left(Z(3)^{\wedge} \theta\right) *(1,2)(3,4)\right]\right) \rightarrow$

Algebra( $\left.\left.\operatorname{GF}(3),\left[\left(Z(3)^{\wedge} \theta\right) *(),\left(Z(3)^{\wedge} \theta\right) *(1,2)\right]\right)\right]$

gap> IsXModAlgebra(CM);

true

gap> Display (CM);

Crossed module [..->...] :-

: Source algebra has generators:

$\left[\left(Z(3)^{\wedge} \theta\right) *()+\left(Z(3)^{\wedge} \theta\right) *(3,4),\left(Z(3)^{\wedge} \theta\right) *(1,2)+\left(Z(3)^{\wedge} \theta\right) *(1,2)(3,4)\right]$

: Range algebra has generators:

$\left[\left(Z(3)^{\wedge} \theta\right) *(),\left(Z(3)^{\wedge} \theta\right) *(1,2)\right]$

: Boundary homomorphism maps source generators to:

$[(Z(3)) *(),(Z(3)) *(1,2)]$

gap> SA3 := SimplicialAlgebraByXModAlgebra(CM);

[AlgebraWithOne $\left(\mathrm{GF}(3),\left[\left(Z(3)^{\wedge} \theta\right) *(1,2),\left(Z(3)^{\wedge} \theta\right) *(3,4)\right]\right) \rightarrow$

Algebra $\left.\left(\mathrm{GF}(3),\left[\left(Z(3)^{\wedge} \theta\right) *(),\left(Z(3)^{\wedge} \theta\right) *(1,2)\right]\right)\right]$

gap> IsSimplicialAlgebra(SA3);

true

\section{ACKNOWLEDGEMENTS}

I would like to thank Zekeriya Arvasi for valuable comments and suggestions.

\section{REFERENCES}

[1] Andrè M. Homologie des Algebrès Commutatives, Springer-Verlag, 206, 1970.

[2] Arvasi Z. Applications in commutative algebra of the Moore complex of a simplicial algebra, $\mathrm{PhD}$ Thesis, University of Wales, Bangor, 1994.

[3] Arvasi Z and Porter T. Freness Conditions for 2-Crossed Modules of Commutative Algebras, Applied Categorical Structures,Vol.6, 455-471, 1998.

[4] Arvasi Z and Ege U. Annihilators, Multipliers and Crossed Modules, Applied Categorical Structures, Vol.11, 487-506, 2003.

[5] Arvasi Z, Kuzpinari TS and Uslu E Ö. Three-crossed modules, Homology Homotopy Appl., no. 2, 161-187, 2009.

[6] Arvasi Z and Odabas A. Crossed Modules of Commutative Algebras and Cat1-algebras in GAP, Version 1.12 Manual for the XModAlg share package for GAP4, 2015, (http://fef.ogu.edu.tr/matbil/aodabas/xmodalg)

[7] Arvasi $\mathrm{Z}$ and Odabas A. Computing 2-dimensional algebras: Crossed modules and Cat1-algebras, Journal of Algebra and Appl. DOI: 10.1142/S0219498816501851, 2016.

[8] Barker M. Representations of Crossed Modules and Cat1-Groups, PhD Thesis, University of Wales, Bangor, 2003. 
Odabaş/ Anadolu Univ. J. of Sci. and Technology A-Appl. Sci. and Eng. 18(1)- 2017

[9] Duskin J. Simplicial Methods and the Interpretation of Triple Cohomology. Memoir A.M.S. Vol. 3 $163,1975$.

[10] GAP - GROUPS, ALGORTIHMS, AND PROGRAMMING, VERSION 4, Lehrstuhl D für Mathematik, RWTH Aachen Germany and School of Mathematical and Computational Sciences, U. St. Andrews, Scotland, 1997.

[11] Gerstenhaber M. On the deformation of rings and algebras, Annals of Mathematics, 84, 1966.

[12] Lichtenbaum S and Schlessinge M. The cotangent complex of a morphism, Transactions of the American Mathematical Society, 128, 41-70, 1967.

[13] Shammu NM Algebraic and Categorical Structure of Category of Crossed Modules of Algebras, Ph.D. Thesis, Osmangazi University, 1992.

[14] Porter T. Some Categorical Results in the Category of Crossed Modules in Commutative Algebra, Journal of Algebra, 109, 415-429, 1987.

[15] Wensley CD, Alp M. Odabas A and Uslu, EÖ. Crossed Modules and cat1-groups in GAP, Version 2.44, Manual for the XMod share package for GAP4, 2015, (http://www.gapsystem.org/Packages/xmod.html)

[16] Whitehead JHC. Combinatorial Homotopy II Bull. Amer. Math. Soc., 55, 453-496, 1949. 